June 18, 2001

Programme overview

\begin{tabular}{|l|l|l|}
\hline Opening session (Aula) & Chair: Ewert Bengtsson & Bo Sundqvist \\
\hline $13.00-13.15$ & Welcome to Uppsala & Steinar Pedersen \\
\hline $13.15-13.25$ & Welcome to a challenging Conference & Lars Engqvist \\
\hline $13.25-14.05$ & $\begin{array}{l}\text { Telemedicine - a tool for a new structure of health care in } \\
\text { Sweden }\end{array}$ & Salah Mandil \\
\hline $14.05-14.30$ & e-Health: its meaning to Development, and its requisites? & \multicolumn{2}{|c|}{ Break } \\
\hline & \multicolumn{2}{|c|}{ Rashid Bashshur } \\
\hline Opening session, cont. & Chair: Bengt Westermark & Masatsugu Tsuji \\
\hline $15.00-15.40$ & On the future of telemedicine & James McGee \\
\hline $15.40-16.00$ & $\begin{array}{l}\text { The Experience and Economic Effects of Tele-Home-Care } \\
\text { in Japan }\end{array}$ & $\begin{array}{l}\text { Telemedicine: A methodology for assuring quality of } \\
\text { performance of health care professionals }\end{array}$ \\
\hline $16.00-16.20$ & $\begin{array}{l}\text { Welcome to most exciting days in Uppsala - all of you will } \\
\text { feel the warmth of our hospitality }\end{array}$ & Hans Kollberg \\
\hline $16.20-16.30$ & \multicolumn{2}{|l|}{ Break } \\
\hline $17.00-20.00$ & ISfT Board Meeting & Host: Bo Sundqvist \\
\hline $20.00-21.00$ & \multicolumn{2}{|l|}{} \\
\hline
\end{tabular}


June 19, 200 Programme overview

\begin{tabular}{l|l} 
Plenary session & TELEMEDICINE AS A TOOL FOR A MORE EQUAL DISTRIBUTION OF HEALTH IN THE WORLD - I
\end{tabular}

(Aula)

$08.00-08.30$

$08.30-08.50$

$08.50-09.10$

\begin{tabular}{|l} 
\\
$09.10-09.30$
\end{tabular}

\begin{tabular}{l}
$09.10-09.30$ \\
\hline
\end{tabular}

Plenary session (Aula)

$10.30-10.50$

$10.50-11.10$

$11.10-12.00$
Chair: Bo Wikström

Telemedicine as a tool for equalising the distribution of healthcare in the world

Virtual reality and robotics in medical education

Technologies for Global Dissemination of Telemedicine:

Mobile communication, ubiquitous computing and

nanotechnology

Electronic patient records - Patient Empowerment: Personal Health Records

Posterviewing, coffee

TELEMEDICINE AS A TOOL FOR A MORE EQUAL DISTRIBUTION OF HEALTH IN THE WORLD - II

Chair: Mehari Gebre-Medhin

World Health Chart, a new enjoyable tool for display of health statistics

Telemedicine - A contributor to improving health systems performance

Can eHealth cross the digital divide?

Pro

Ricky Richardson
Peter J Buxton

Christoph R. Kaufmann

Richard M. Satava

Peter Waegemann

Hans Rosling

Orvill Adams

A pro/con discussion lead by Hans Kollberg

Con

Benedict Stanberry
Telemedicine as a too for a more equitable distribution of health care delivery around the world*

(satellite conferences and demonstrations)

Chair: Stefan Cajander Co-chair: André Morin

Room: X

Live conferences with WHO, BDT-IUT, EC, G8, Tunis

Illustrations of telemedicine practices in Developing Countries: Tunis, Dakar, New Delhi, Buenos Aires

DialogVision: Global

Telemedicine Pilot Project (Uppsala - Tunis)

*see separate programme 


\begin{tabular}{|c|c|c|c|c|}
\hline \multirow[t]{8}{*}{$\begin{array}{c}\text { Parallel sessions } \\
13.30-15.00\end{array}$} & $\begin{array}{l}\text { Major incidents. The } \\
\text { Past, Present and } \\
\text { Future of Training and } \\
\text { Care } \\
\text { Chair: Peter Buxton } \\
\text { Co-chair: Lars Lundberg } \\
\text { Aula }\end{array}$ & $\begin{array}{l}\text { Electronic patient } \\
\text { record } \\
\text { Chair: Pål Lindström } \\
\text { Room: IV }\end{array}$ & $\begin{array}{l}\text { Telemedicine in the } \\
\text { Baltic and Arctic } \\
\text { regions } \\
\text { Chair: Siri Uldal } \\
\text { Co-chair: Urban Pontén } \\
\text { Room: IX }\end{array}$ & \multirow[t]{15}{*}{ 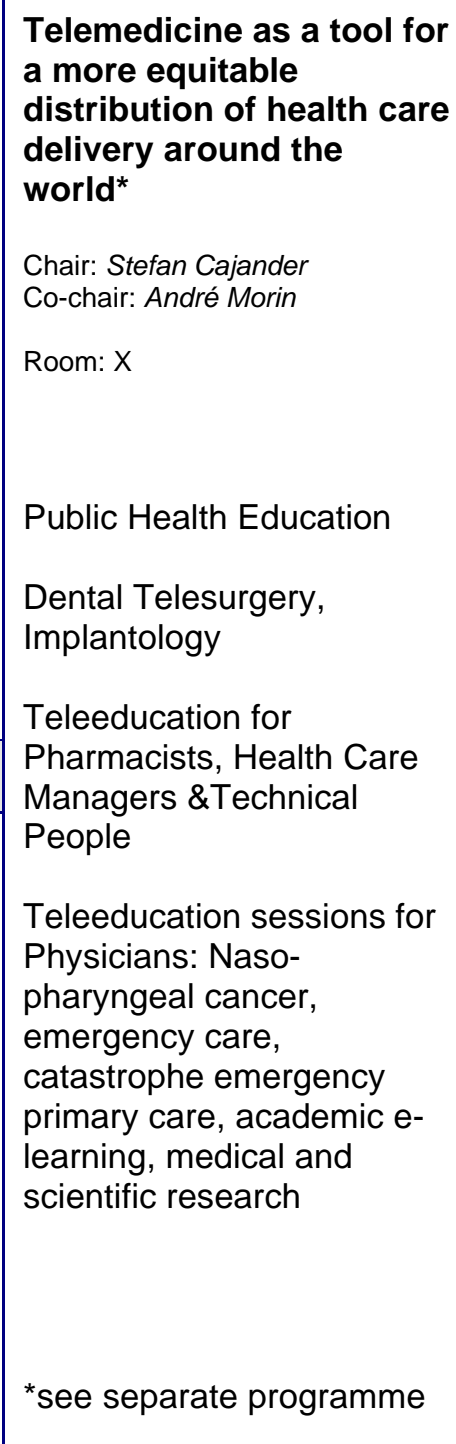 } \\
\hline & OP01 & OP05 & OP10 & \\
\hline & OP02 & OP06 & OP11 & \\
\hline & OP03 & OP07 & OP12 & \\
\hline & OP04 & OP08 & OP13 & \\
\hline & & OP09 & OP14 & \\
\hline & & & OP15 & \\
\hline & \multicolumn{3}{|c|}{ Exhibition } & \\
\hline \multirow[t]{7}{*}{$\begin{array}{l}\text { Parallel sessions } \\
16.00-17.30\end{array}$} & $\begin{array}{l}\text { Virtual reality, trauma } \\
\text { and ambulance } \\
\text { Chair: Mats-Ola Svensson } \\
\text { Aula }\end{array}$ & $\begin{array}{l}\text { Teledentistry } \\
\text { Chair: Kenneth Eaton } \\
\text { Co-chair: Sabine Koch } \\
\text { Room: IV }\end{array}$ & $\begin{array}{l}\text { Telemedicine in } \\
\text { remote areas } \\
\text { Chair: Steinar Pedersen } \\
\text { Room: IX }\end{array}$ & \\
\hline & $\mathrm{PO} 22$ & OP18 & OP24 & \\
\hline & OP16 & OP19 & OP25 & \\
\hline & OP17 & OP20 & OP26 & \\
\hline & Live video demonstration & OP21 & OP27 & \\
\hline & & OP22 & OP28 & \\
\hline & & OP23 & OP29 & \\
\hline
\end{tabular}





\section{June 20, 2001}

Early bird session

(Aula)

$07.15-07.45$

(coffee will be served)

(Pinary session (Aula)

Plenary session (Aula)

$$
08.00-09.30
$$

\begin{tabular}{|l}
\hline \\
\hline \\
\hline \\
\end{tabular}

Plenary session (Aula)

$$
10.30-12.00
$$

\begin{tabular}{|l|l} 
& Chair: Anders Fagerlund \\
\hline & A live video demonstration of a clinical round with an \\
expert panel from the university hospital supporting the
\end{tabular}

expert panel from the university hospital supporting the

staff and patients at a distant district hospital

\section{Traumasupport}

Baltic International Telemedicine NETwork - the BIT NET Project

Sjunet - the IT-infrastructure for healthcare

Neurophysiology in the National IP-net

Transparancy - a non negotiable ethic in working with patients
Real time contact with Hong-Kong

Break

Laurel Simmons

Teuvo Peltoniemi

Lennart Magnusson

Posterviewing, coffee

FICTION. (TELEMEDICINE IN UPPSALA AND SWEDEN)

Stefan Cajander, Maggie Granstrand, Inga Sjöberg, Rose-Maria Toffia, Nils-Olof Gustavsson \& Ulf Oskarsson

Claes Juhlin, Pjotr Tracz

Anders Fagerlund, Uwe Ewald, Urban Pontén

KG Nerander

Roland Flink

Angelica Frithiof

Lunch 


\begin{tabular}{|c|c|c|c|c|c|}
\hline \multirow[t]{7}{*}{$\begin{array}{c}\text { Parallel sessions } \\
13.30-15.00\end{array}$} & $\begin{array}{l}\text { Telemedicine - a tool } \\
\text { for global education } \\
\text { Chair: Leif Hambraeus } \\
\text { Co-chair: T. Tylleskär } \\
\text { Aula }\end{array}$ & $\begin{array}{l}\text { Telepsychiatry } \\
\text { Chair: Russel D'Souza } \\
\text { Co-chair: Frits-Axel Wiesel } \\
\text { Room: IV }\end{array}$ & $\begin{array}{l}\text { Quality assurance } \\
\text { Chair: Magnus Hjelm } \\
\text { Co-chair: A. Frithiof } \\
\text { Room: IX }\end{array}$ & $\begin{array}{l}\text { Organisational and } \\
\text { juridical aspects } \\
\text { Chair: Leif Erik Nohr } \\
\text { Co-chair: Bo Wikström } \\
\text { Room: XI }\end{array}$ & $\begin{array}{l}\text { Telepathology } \\
\text { Chair: Stefan Cajander } \\
\text { Co-chair: Ronald Weinstein } \\
\text { Room: X }\end{array}$ \\
\hline & OP30 & OP36 & OP42 & OP48 & OP54 \\
\hline & OP31 & OP37 & OP43 & OP49 & OP55 \\
\hline & OP32 & OP38 & OP44 & OP50 & OP56 \\
\hline & OP33 & OP39 & OP45 & OP51 & OP57 \\
\hline & OP34 & OP40 & OP46 & OP52 & \\
\hline & OP35 & OP41 & & OP53 & \\
\hline \multirow{8}{*}{$\begin{array}{c}\text { Parallel sessions } \\
16.00-17.30\end{array}$} & \multicolumn{5}{|c|}{ Exhibition } \\
\hline & $\begin{array}{l}\text { Web based healthcare } \\
\text { and education } \\
\text { Chair: Göran Petterson } \\
\text { Co-chair: Jonas Boberg } \\
\text { Aula }\end{array}$ & $\begin{array}{l}\text { Teleradiology and } \\
\text {-cardiology } \\
\text { Chair: Olof Jarlman } \\
\text { Room: IV }\end{array}$ & $\begin{array}{l}\text { Tele homecare } \\
\text { Chair: R. Richardson } \\
\text { Co-chair: E. Rylander } \\
\text { Room: IX }\end{array}$ & $\begin{array}{l}\text { Administration and } \\
\text { economics } \\
\text { Chair: Björn-Erik Erlandson } \\
\text { Room: XI }\end{array}$ & $\begin{array}{l}\text { Telepathology } \\
\text { Chair: Stefan Cajander } \\
\text { Co-chair: Ronald Weinstein } \\
\text { Room: X }\end{array}$ \\
\hline & OP61 & OP67 & OP73 & OP79 & OP58 \\
\hline & OP62 & OP68 & OP74 & OP80 & OP59 \\
\hline & OP63 & OP69 & OP75 & OP81 & OP60 \\
\hline & OP64 & OP70 & OP76 & OP82 & \\
\hline & OP65 & OP71 & OP77 & OP83 & \\
\hline & OP66 & OP72 & OP78 & OP84 & \\
\hline
\end{tabular}




\begin{tabular}{|c|c|c|}
\hline June 21, 2001 & \multicolumn{2}{|l|}{ Programme overview } \\
\hline \multirow{2}{*}{$\begin{array}{l}\text { Plenary session } \\
\text { (Aula) }\end{array}$} & \multicolumn{2}{|l|}{ HOW WILL WE PROCEED } \\
\hline & \multicolumn{2}{|l|}{ Chair: Leif Karlsson } \\
\hline \multirow[t]{2}{*}{$09.00-10.00$} & Copyright vs Community in the age of computer networks & Richard Stallman \\
\hline & \multicolumn{2}{|c|}{ Refreshments } \\
\hline $10.30-10.45$ & eHealth: The Road Ahead. Where is Europe placed? & Ricky Richardson \\
\hline $11.00-11.15$ & Organizational aspects of telemedicine & Henrik Linderoth \\
\hline \multirow[t]{2}{*}{$11.15-11.30$} & & Gudjon Magnusson \\
\hline & \multicolumn{2}{|c|}{ Short break } \\
\hline Plenary session & \multicolumn{2}{|l|}{ END OF ENCHANTING DAYS } \\
\hline $12.30-12.45$ & Summing up & Hans Kollberg, Björn-Erik Erlandsson \\
\hline $12.45-12.50$ & Welcome to Germany & Michael Nerlich \\
\hline $12.50-13.00$ & $\begin{array}{l}\text { Closing of the } 6 \text { th International Conference on the Medical } \\
\text { Aspects of Telemedicine }\end{array}$ & Steinar Pedersen \\
\hline
\end{tabular}

